\title{
Position tracking using inertial and magnetic sensing aided by permanent magnet
}

\author{
Michał Meina \\ Department of Informatics, \\ Faculty of Physics, Astronomy and Inf. \\ Nicolaus Copernicus University, \\ Grudziadzka 5, 87-100 Toruń, Poland
}

\author{
Krzysztof Rykaczewski \\ Faculty of Mathematics and Comp. Science, \\ Nicolaus Copernicus University, \\ Chopina 12/18, 87-100 Toruń, Poland
}

\author{
Andrzej Rutkowski \\ Faculty of Mathematics and Comp. Science, \\ Nicolaus Copernicus University, \\ Chopina 12/18, 87-100 Toruń, Poland
}

\begin{abstract}
This paper describes a method for spatial tracking of a strapdown device that can be used for design of humancomputer interfaces. Inertial Measurement Unit (IMU) is used to obtain 6-dof position exploiting the so-called ZUPT technique by the means of the Kalman Filter. Additional corrections of position are done using magnetometer readings in the presence of static magnetic field induced by permanent magnet that overshadow geomagnetic field. This correction allows us to overcome drifting errors of integration of IMU readings. We have also presented comparisons of different models for magnetic field reconstruction that is crucial for this system.
\end{abstract}

\section{INTRODUCTION}

H UMAN-Computer interfaces that uses hand gestures, object manipulation or any other pervasive technology always require position tracking subsystem. Recent developments in micro-electro mechanical systems (MEMS) enables researchers to build new wearable, position aware devices by utilization of miniaturized and low-power sensing devices. For example, inertial sensors are commonly used for construction of 6-dof (orientation and position) spatial tracking systems [1], [2]. Nevertheless, precision of such sensing devices is still very low, therefore it is needed to adopt more complex computations and signal filtering techniques. In this paper, we present an inertial position tracking system that uses additional correction by positioning in magnetic field induced by permanent magnet. Previous works exploited an array of magnetic sensors to unambiguously estimate position, while we propose a data fusion algorithm that uses just one magnetic sensor along with inertial sensor.

Data fusion algorithms are used commonly with inertial devices - in order to estimate orientation of the sensor, stateof-the art filter [3] integrate angular rate (obtained from gyroscope) in order to calculate rough quaternion rates and then uses gravitational force (obtained by accelerometer) to correct roll and pitch angles. Finally, magnetometer readings are exploited to correct yaw angle. The best known algorithm that can be used in this setup for position estimation exploits the so-called "zero-velocity" update (ZUPT). Second integral of acceleration outputs position with accumulative error. Additional statistics are used to test if the sensor is still-velocity, therefore, should be zero (most of the time it is not and one can use this information to correct position). This technique was proposed in [4] for inertial navigation using foot-mounted inertial measurement unit. We have extended the technique introducing Magnetic Update (MUPT) that introduces small correction of position if the sensor is exposed to magnetic field. In presented setup properties of magnetic field must be known beforehand, basing on extensive calibration procedure.

Contribution of this paper is as follow: (1) design and evaluation of position tracking system by fusion of inertial and magnetic sensing in the presence of magnetic field induced by permanent magnet, (2) evaluation of magnetic field reconstruction techniques, (3) positioning algorithm in that use

\section{RELATED WORK}

Measurements of magnetic field have long been used in the problem of spatial tracking. Starting with the problem of locating buried magnet, one solution [5] used measurements of magnetic field generated by beacon device. In 1979 Raab et al. [6] proposed a complete system for relative position and orientation tracking using active 3-axis magnetic dipole source. Technique was further described and analysed on simulated data by Raab [7]. Similar methods are now used in commercial tracking solutions like Polhemus Fastrack or gaming controller Razer Hydra. Although these methods are useful in some situations, power requirements and high weight of magnetic coils disqualify it in context of wearable devices, with exception of specific usages as described in [8]. Since the advent of MEMS inertial and magnetic sensors, many solutions have been proposed for problem of position and orientation tracking using fusion of inertial and passive magnetic measurements [9], [10], [11]. Unfortunately, inertial systems suffer from systematic error accumulation. It is common [12], [13] to address this problem with ZUPT, as proposed in [4]. Although, as pointed out in [14], this solution suffers from many drawbacks and its usefulness is limited.

Geomagnetic field measurements are also susceptible to errors such as interference from ferromagnetic mass and electric devices. Zachmann tried correcting magnetic measurements by gathering calibration samples of magnetic field in a volume of tracking space [15]. Some authors tried using these distortions for detection and low accuracy tracking of metallic objects [16], [17]. Magnetic field produced by permanent magnets locally overshadow earth's, thus extensive research have been done on reverse problem of tracking permanently 
magnetised markers with relation to magnetometer and (most often) arrays of magnetometers [18], [19], [20], [21].

Finally, in 2015 Kortier et al. [22] explored a system for tracking neodymium magnet paired with accelerometer and gyroscope with respect to array of four magnetometers in parallel with inertial sensor.

We propose a system where magnetometer is coupled with inertial sensors in a package and tracking is carried out with respect to neodymium magnet.

\section{Modelling Vector Field Of A Permanent MAGNET}

In order to obtain the position of magnetic sensor in the presence of static magnetic field the straightforward idea is to use a model of the magnetic field and match the empirical measurement (obtained by magnetometers) to the theoretical one. The idea is to construct error function that will be minimized in order to find position of the sensor (or array of the sensors). This idea was investigated in many papers, e.g. [19], [21]. The technique requires finding a method for determining theoretical value of magnetic vector field.

More formally, we are searching for a function $P$ such that

$$
P_{\Theta_{s}^{m}}(x, y, z)=\vec{B}
$$

where $\vec{B}$ is a vector of magnetic induction in given position $(x, y, z) \in \mathbb{R}^{3}$ and $\Theta_{s}^{m}$ is an orientation of the sensing device $s$ in the magnet frame of reference $m$ (one can understand it as a transformation from sensor frame of reference to magnet frame of reference).

Magnetic induction vector in our case consists of three components: geomagnetic field $\vec{B}_{\text {geo }}$, field of permanent magnet $\vec{B}_{\text {mag and ferromagnetic part considered as environment noise }}$ $\vec{B}_{\text {env }}$. Therefore,

$$
\vec{B}=\vec{B}_{\text {mag }}+\vec{B}_{\text {geo }}+\vec{B}_{\mathrm{env}}
$$

For strong magnets, however, we can omit environmental and geomagnetic component, hence $\vec{B} \approx \vec{B}_{\text {mag }}$.

The model of the magnetic field is difficult to calculate in real time, so we are looking for some approximation of it. For this reason, we have investigated and compared different techniques: (1) dipole model, (2) integration from Maxwell equations using finite element method (FEM) simulators, and (3) field interpolation on empirical (and sparse) sampling. These methods are described below.

\section{A. The Magnetic Dipole}

Magnetic field of strong magnet can be approximated using magnetic dipole model [23]:

$$
\vec{B}_{\vec{\mu}}(\vec{r})=\frac{1}{\|\vec{r}\|^{5}}\left(3(\vec{\mu} \vec{r}) \vec{r}-\vec{\mu}\|\vec{r}\|^{2}\right),
$$

where $\mu$ is a magnetic dipole moment (that characterize magnetic field source) and $\vec{r}$ is a vector from the magnetic dipole source to the observation point. It is important to note that, since we assume that magnetic field source is infinitely small point, this model is accurate only when $\|\vec{r}\| \gg r_{m}$ (where $r_{m}$ is a size of magnet).

We can rewrite Eq. (3) into components to obtain formulas that characterize components of $\vec{B}=:\left[B_{x}, B_{y}, B_{z}\right]$ :

$$
\begin{aligned}
& B_{x}=3|\mu| \frac{z y}{\left(x^{2}+y^{2}+z^{2}\right)^{\frac{5}{2}}}, \\
& B_{y}=3|\mu| \frac{z x}{\left(x^{2}+y^{2}+z^{2}\right)^{\frac{5}{2}}}, \\
& B_{z}=|\mu| \frac{2\left(x^{2}-y^{2}\right)-z^{2}}{\left(x^{2}+y^{2}+z^{2}\right)^{\frac{5}{2}}} .
\end{aligned}
$$

From Equation (4) we see that in order to use the above model, we must carry out a calibration of the magnet (find the magnetic dipole moment $\mu$ for a particular magnet). This characteristic is included in the documentation that came with the magnet, nevertheless, we have found this parameter in the experimental section by defining an error function and making its optimization. In order to find this parameter, only one measurement in some known position is needed.

Usage of magnetic dipole moment for defining position estimation error function enables us to carry out the gradient function in analytical form. That makes minimization very fast.

\section{B. Magnetostatic simulation using FEM}

FEM (Finite Element Method) is a method of integrating differential equation used to find approximate solutions of many physical models. In our context it is used to solve the Maxwell equations. Magnetic dipole moment, which is a parameter for this FEM model, was calculated by experiment. The boundary conditions were chosen at far distance from field source in the manner that changing them will not affect the magnetic field in region of interest.

We used QuickField ${ }^{1}$ library, that outputs the exact solution at some points and interpolates the rest. The position of the points are optimized.

\section{Interpolation of a vector field}

In this section, we propose a fast method to approximate the magnetic field using empirical readings-our magnetic field calibration procedure.

The method described below is based on measurements of magnetic field $\vec{B}$ at predetermined positions in the vicinity of the magnet, and then determining the interpolating function for any point in the area of interest $D$. For this purpose, we used the Delaunay triangulation (DT). The idea of this method is to find simplex, inside which there is a point of interest, and then counting the weighted sum of the vertices of the simplex (on which empirical measurement is known).

More formally, suppose we have made $n$ measurements of magnetic field $\hat{\vec{B}}\left(p_{i}\right), i=1, \ldots, n$, in points $p_{i}:=\left(x_{i}, y_{i}, z_{i}\right)$, $i=1, \ldots, n$.

Let us consider the division of space $\mathbb{R}^{d}$ on $(d+1)$-simplices $\left\{\sigma_{j}:=\sigma\left(\left\{p_{i_{k}}\right\}_{k=0}^{K_{j}}\right)\right\}_{j \in J}$, where $\sigma\left(\left\{p_{l}\right\}_{l=1}^{L}\right)$ is the simplex determined by vertices $p_{l}, l=1, \ldots, L$, such that:

- for $k \neq l, k, l \in J$, intersection of simplices $\sigma_{k} \cap \sigma_{l}$ is either a common wall of $\sigma_{k}$ and $\sigma_{l}$, or is empty,

${ }^{1}$ http://www.quickfield.com/ 
- the interior of the circumscribed sphere on any simplex $\sigma_{j}$ does not contain any point $\left\{p_{i}\right\}_{i=1}^{n}$.

Our area of interest $D$ is the sum of all the above simplices. In the present context we only consider the division of $\mathbb{R}^{2}$ and $\mathbb{R}^{3}$, therefore, we can informally say that Delaunay triangulation divides the space into triangles and tetrahedrons, respectively, such that none of these objects is deformed (i.e. do not contain "very sharp" angles). A popular method for determining triangulation is based on the dual space (we show an example for $\left.\mathbb{R}^{2}\right)$ : we consider the projection $\left(p_{x}, p_{y}\right) \mapsto\left(p_{x}, p_{y}, p_{x}^{2}+p_{y}^{2}\right)$ and then calculate the convex hull of this set of points. Then the corresponding points will create DT.

Let $\vec{B}_{D T}(p)$ denotes the magnetic induction vector calculated by Delaunay interpolation at point $p$ contained in the convex hull of $p_{i}$. For determining $\vec{B}_{D T}$ DT uses barycentric coordinates $\left\{\lambda_{k}\right\}_{k=1}^{d+1}$ of $p$ with respect to $\left\{p_{i_{k}}\right\}_{k=1}^{d+1}$. In conclusion, we can represent it as a linear combination

$$
\hat{\vec{B}}_{D T}(p)=\sum_{k=0}^{d+1} \lambda_{k} \hat{\vec{B}}\left(p_{i_{k}}\right)
$$

such that $\sum_{k=1}^{d+1} \lambda_{k}=1$ and $\lambda_{1}, \ldots, \lambda_{d+1} \geq 0$, where points $p_{i_{k}}$ are vertices of simplex $\sigma$ such that $p \in \sigma$. It is worth noting that calculation of coefficients $\lambda_{k}$ for point $p=\left(p_{x}, p_{y}\right)$ in $\mathbb{R}^{2}$ (and, analogically, in $\mathbb{R}^{3}$ ) using reference points $p^{1}, p^{2}, p^{3}$, is limited to determining the solution of the following system of equations

$$
\left[\begin{array}{ccc}
p_{x}^{1} & p_{x}^{2} & p_{x}^{3} \\
p_{y}^{1} & p_{y}^{2} & p_{y}^{3} \\
1 & 1 & 1
\end{array}\right]\left[\begin{array}{c}
\lambda_{1} \\
\lambda_{2} \\
\lambda_{3}
\end{array}\right]=\left[\begin{array}{c}
p_{x} \\
p_{y} \\
1
\end{array}\right]
$$

From the computational complexity point of view, the most expensive operation is finding a simplex, inside which the point is located. To do this, one can apply a hierarchical decomposition of simplices, which represents collection of simplices in the form of binary search tree. Asymptotic cost of finding simplex is, therefore, $\mathcal{O}(\log n)$, however, because of need for preprocessing and higher storage requirements, such representation may be not suitable for implementation on small microprocessors. Best alternative seems to be a classic walking algorithm [24]. As noted in [25], Mücke shows [26] that careful use of walking algorithm can bring down expected time close to $\mathcal{O}\left(n^{1 / 4}\right)$ (for $d=3$ ).

\section{Magnetic Field Reconstruction}

In order to check its reliability our methodology was as follows: we recorded vectors of magnetic induction in many places on common plane and compared it with the theoretical model. In this section, we will present experimental result of reconstruction of magnetic field.

The magnetic field was generated by arrangement of three cylindrical neodymium magnets $(\oslash=22[\mathrm{~mm}], h=10[\mathrm{~mm}]$, direction of magnetization was along shorter axis). According to specification, at the distance of $0.7[\mathrm{~mm}]$ in the direction of magnetization the magnetic field strength was $\approx 0.380[\mathrm{~T}]$. Measurement was performed with MEMS magnetometer LSM303D, that is able to measure magnetic field of maximum

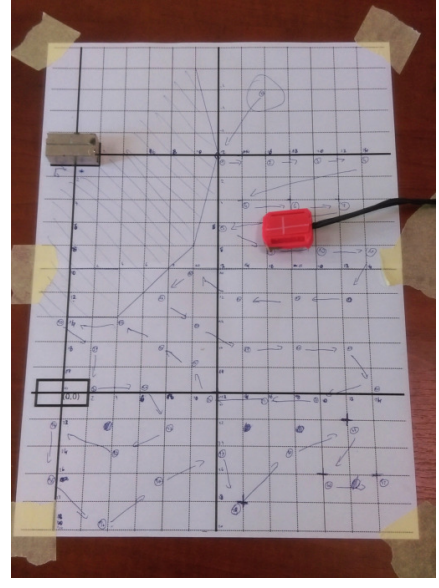

Fig. 1: Experimental setup.

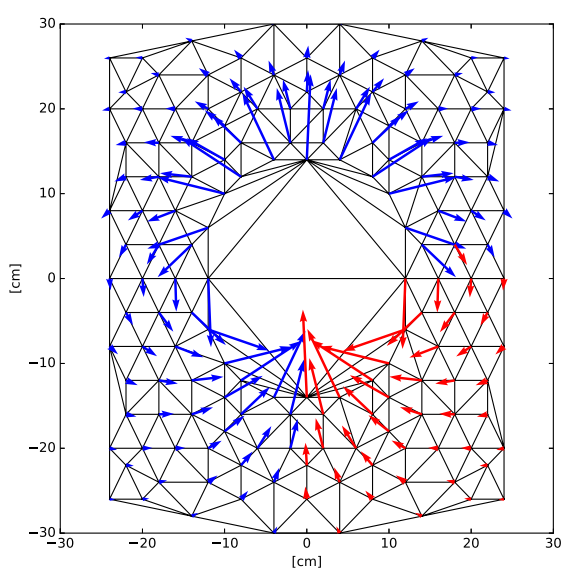

Fig. 2: Magnetic field by Delaunay triangulation. Red arrows depicts empirical measurements whereas blue arrows represent symmetrical reflection of those.

strength of $\pm 1.2[\mathrm{mT}]$ with 16 bits resolution (values with 16 meaningful bits). See Fig. 1 for our experimental setup.

At the beginning, we checked properties of the field with magnets arranged in the above manner.

We've done several experiments, in order to show that magnetic field around the cylinders is symmetrical. Moreover, several experiments have been performed to check the accuracy of different models. Geomagnetic field was measured beforehand and discarded from both calibration dataset and test dataset. Experimental station is illustrated in Fig. 1. Table III-D shows the results of different tests.

Test 1 was divided into several subtest: Iq, IIq, IIIq, IVq, $r<20, r>=20$. It consists of measurements when magnet was not rotated.

Test 2, 3 and 4 consists of measurements with magnet rotated along magnetization axis by 90,180 and 270 degrees, respectively, without changing its location. Each test consisted of 54 measurements in places which were different than 
TABLE I: Comparison of magnetic field reconstruction using different models.

\begin{tabular}{|c|c|c|c|c|c|c|}
\hline & dipole & $\begin{array}{c}\measuredangle \overrightarrow{B_{e}} \overrightarrow{B_{m}}[\mathrm{deg}] \\
\text { fem }\end{array}$ & interpolant & dipole & $\begin{array}{c}10^{-2}|B| \text { [gauss] } \\
\text { fem }\end{array}$ & interpolant \\
\hline test $1 / 0^{\circ}$ & $19.37 \pm 9.28$ & $7.89 \pm 4.96$ & $3.62 \pm 3.57$ & $72.17 \pm 72.09$ & $113.71 \pm 50.49$ & $14.15 \pm 16.11$ \\
\hline $\mathrm{Iq}$ & $17.96 \pm 9.12$ & $7.67 \pm 4.71$ & $1.62 \pm 1.32$ & $65.16 \pm 55.32$ & $127.09 \pm 62.41$ & $5.62 \pm 7.94$ \\
\hline IIq & $22.81 \pm 8.03$ & $10.40 \pm 4.41$ & $5.82 \pm 3.81$ & $59.31 \pm 56.11$ & $108.68 \pm 23.94$ & $15.22 \pm 19.41$ \\
\hline IIIq & $20.57 \pm 9.29$ & $7.68 \pm 5.24$ & $3.99 \pm 4.78$ & $70.38 \pm 63.26$ & $111.28 \pm 50.20$ & $20.91 \pm 19.18$ \\
\hline IVq & $18.70 \pm 8.15$ & $6.41 \pm 4.57$ & $3.15 \pm 2.10$ & $58.69 \pm 54.25$ & $124.10 \pm 39.39$ & $11.07 \pm 6.31$ \\
\hline $\mathrm{r}<20$ & $23.09 \pm 10.74$ & $11.69 \pm 5.02$ & $2.67 \pm 1.99$ & $128.76 \pm 91.99$ & $122.44 \pm 75.47$ & $25.12 \pm 22.40$ \\
\hline$r>=20$ & $17.52 \pm 7.83$ & $5.99 \pm 3.66$ & $4.09 \pm 4.06$ & $43.88 \pm 34.11$ & $109.34 \pm 30.30$ & $8.66 \pm 6.95$ \\
\hline test $2 / 90^{\circ}$ & $19.46 \pm 9.50$ & $8.24 \pm 5.31$ & $4.07 \pm 3.60$ & $72.07 \pm 72.16$ & $113.55 \pm 51.35$ & $14.06 \pm 15.79$ \\
\hline test $3 / 180^{\circ}$ & $19.31 \pm 9.24$ & $7.98 \pm 4.75$ & $3.70 \pm 3.64$ & $72.08 \pm 71.99$ & $114.08 \pm 51.12$ & $13.56 \pm 14.43$ \\
\hline test $4 / 270^{\circ}$ & $19.08 \pm 9.21$ & $7.63 \pm 5.05$ & $3.77 \pm 3.56$ & $71.92 \pm 71.63$ & $113.67 \pm 50.58$ & $15.53 \pm 17.49$ \\
\hline
\end{tabular}

calibration points. Since magnetometer has maximum range of measurements, readings were taken at distances of less that $12[\mathrm{~cm}]$ from the magnet.

Left part of the table shows angle of deviation between model magnetic field $\vec{B}_{m}$ and experimentally measured magnetic field $\vec{B}_{e}$. Right part of the table shows means and standard deviations of norms between $\vec{B}_{m}$ and $\vec{B}_{e}$ (i.e. | $\left.\left\|\vec{B}_{m}\right\|-\left\|\vec{B}_{e}\right\| \mid\right)$.

In Table III-D one can see that error is similar in each model, indicating that the measured magnetic field is indeed symmetrical along the axis of magnetization. Differences between quarters of the coordinate system are larger, but this is probably due to inaccuracies of measurement. Dipole model (as expected) makes smaller error (in particular, when it comes to the norm of $\vec{B}$ ) when we measure at distant places $(r \geq 20[\mathrm{~cm}])$ from the source field. Field calculated using FEM is, roughly speaking, two times better than the dipole model. Our method was marked as "interpolant" and is about five times better that dipole and about 2 times better that FEM.

Bearing in mind the above, we assume that around cylindrical magnetic the field is symmetrical, therefore calibration measurements were taken only on the one selected quadrant of a plane.

Delaunay triangulation is depicted in Fig. 2. We see there 42 measurements of $\vec{B}$ (depicted as red arrows) and 115 vectors (blue arrows) that were derived using symmetrical reflection of those 42 .

\section{POSITIONING IN EMPIRICALLY INTERPOLATED MAGNETIC FIELD}

Positioning within the magnetic field using a magnetometer is usually performed using the layout of sensors and the dipole model. Dipole model considered for its own sake is biased by error. However, it can be compensated by using more readings and their relative positions. Additionally, a more accurate position can be determined with a greater number of sensors.

Therefore, in order to calculate the precise position it is usually necessary to use at least two sensors. However, in the paper we want to get rid of this constraint and estimate the position with a single magnetometer in the magnetic field. Hence, we cannot mitigate errors of the model by simultaneous readings and, therefore, we can find only one parameter: either the location (having a fixed orientation) or the orientation (having a fixed position). For that we will minimize the cost function defined as follows

$$
f_{\vec{B}_{e}}(p)=\left|\vec{B}_{m}(p)-\vec{B}_{e}\right|^{2}+\left(\left|\vec{B}_{m}(p)\right|-\left|\vec{B}_{e}\right|\right)^{2},
$$

where $\vec{B}_{e}$ is experimentally measured magnetic field and $\vec{B}_{m}(p)$ is the value of model magnetic field at point $p$. Calculation of $\vec{B}_{m}(p)$ is the most computationally expensive operation.

Since this function is convex in a neighbourhood of current position, we know that (locally) the minimum value of function (7) is uniquely determined. As it was stated above, in the presence of at least two sensors we can use dipole model. Therefore, error function is smooth and minimization can be done by gradient descent algorithm which it terminates after just a few iterations. In the case of our model, the error function is created from DT and since the data is collected empirically we do not have smoothness any more. That is why we use nongradient methods, e.g. Nelder-Mead. This algorithm also terminates after few iterations, but it needs many more error function evaluations.

In Fig. 3 we show the magnitudes of error function (Eq. 7) with respect to number of evaluations during the execution of minimization, starting from two initial positions. For each minimization these were selected by first randomly choosing known destination point and then selecting random position from its neighbourhood within the range of $1[\mathrm{~cm}]$ and $4[\mathrm{~cm}]$, respectively. The thick line on the chart indicates the average and is surrounded by a plot of the standard deviation at each iteration.

\section{FUSION OF INERTIAL POSITIONING AND MAGNETIC}

The fusion algorithm is implemented by the means of Kalman Filter which estimates the error state vector $\delta x_{k}=$ $\left[\begin{array}{lll}\delta v_{k} & \delta p_{k} & \delta C_{k}\end{array}\right]$, which represents velocity, position and orientation (roll, pitch, yaw) errors. Details of the algorithm are described in Alg. 1-as an input it takes readings from IMU $(a, \omega, m-$ which are acceleration, angular rates and magnetic induction vector respectively). 


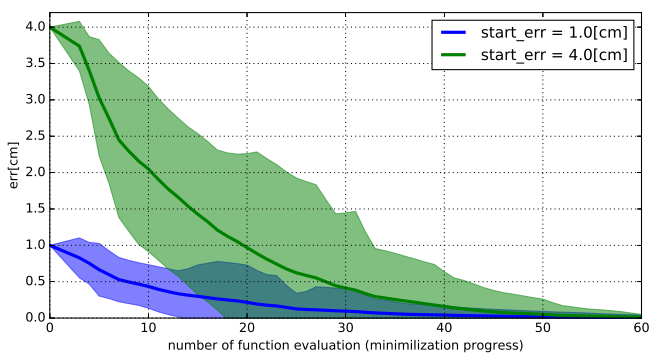

Fig. 3: Converge of minimization.

State transition matrix $F_{k}$ is defined as follow:

$$
F_{k}:=\left[\begin{array}{ccc}
I_{3 \times 3} & \Delta t I_{3 \times 3} & 0_{3 \times 3} \\
0_{3 \times 3} & I_{3 \times 3} & 0_{3 \times 3} \\
\Delta t S_{k} & 0_{3 \times 3} & I_{3 \times 3}
\end{array}\right],
$$

where $S_{k}$ is a skew-symmetric cross-product operator matrix given by:

$$
S_{k}:=\left[\begin{array}{ccc}
0 & -a_{z, k}^{N} & a_{y, k}^{N} \\
a_{z, k}^{N} & 0 & -a_{x, k}^{N} \\
-a_{y, k}^{N} & a_{x, k}^{N} & 0
\end{array}\right]
$$

The $S_{k}$ matrix is used for accumulating orientation errors with respect to velocity error estimates. This method is inspired by technique called "alignment transfer" [27] and was introduced to ZUPT-based inertial navigation systems by Foxlin [4]. In our algorithm we updated the orientation estimation from original Foxlin work by usage of complementary filter (line 6) which was developed by Madgwick [3]. In our previous work [28] we showed his setup works better for orientation estimation for the object in motion with zupt-based filters.

At line 11 two statistical test are performed: one for zero velocity hypothesis testing (IsZUPT) and one for position estimation error test (ISMUPT). For the zero-velocity hypothesis testing we apply the following formula at $k$-th reading:

$$
\operatorname{Var}\left(\left\{\sqrt{\omega_{x}^{2}(t)+\omega_{y}^{2}(t)+\omega_{z}^{2}(t)} \mid t=k-w, \ldots, k\right\}\right)<\epsilon_{1}
$$

Simply speaking Eq. 10 is a windowing $w$-length function that checks if variance of gyroscope readings do not exceed certain threshold $\left(\epsilon_{1}\right)$. For zero-velocity hypothesis testing there exist lots of solution in the literature (see [29], [30], [31])

In order to check if the correction based on magnetic positioning observation need to be performed another test is executed: IsMUPT. This test checks if covariance error didn't exceed certain threshold:

$$
\sum_{i=3}^{6}\left(P_{i \times i}\right)<\epsilon_{2}
$$

Eventually at lines 19-23 the Kalman Update is performed basing on two version of observation matrix $H$; one for velocity pseudo-observation update (line 13) and second one or true position observation (line 16).

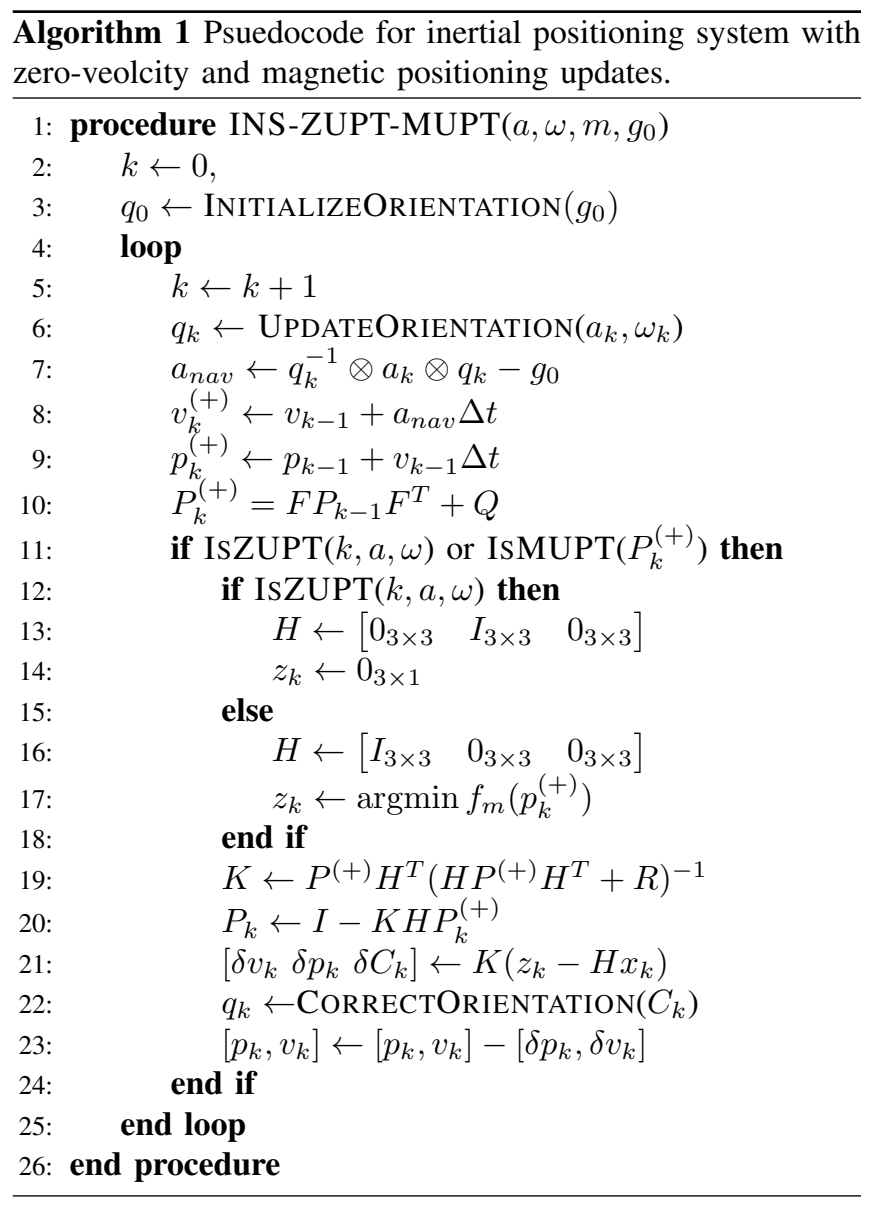

\section{EXPERIMENTAL RESULTS}

Experiment was conducted using sensor and a magnet, which properties were described in section III-D. The sensor was moved freely over the table with moderate speed in various trajectories (with and without still phases). The true path was obtained using optical system, composed from two cameras that were observing diode mounted on top of the sensor. Example of such movement is depicted on the Fig. 4. Fig. $4 \mathrm{~b}$ depicts trajectories computed by algorithm described in previous section (blue lines), whereas Fig. 4a shows the same algorithm in which no magnetic update has been made. Note that path is depicted only between zero-velocity phasesthe a posterori states. The a priori states are depicted by the dotted line as "intermediate states". The read arrows indicates magnetic field measurements done at particular place and the green line is the true path observed by optical system. As a visual aid magnetic field was enclosed using interpolation method-black field lines.

Error drift in inertial navigation system describes the situation where there is a random inaccuracy introduced at each step of computation. It source lays in (1) sensor error and (2) floating-point computation. It is very challenging (or impossible) to model this error, therefore there is no method for position tracking basing only on IMU readings. 


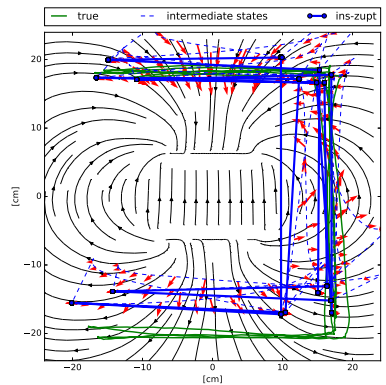

(a) Inertial System with zerovelocity updates.

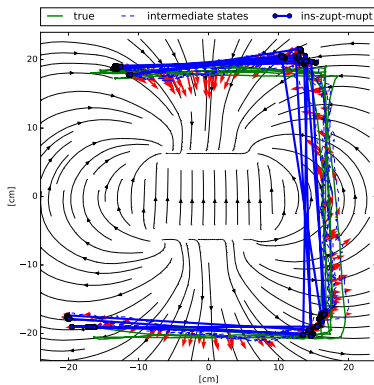

(b) Inertial system with zerovelocity updates and magnetic positioning.
Fig. 4: Comparison of position tracking algorithms.

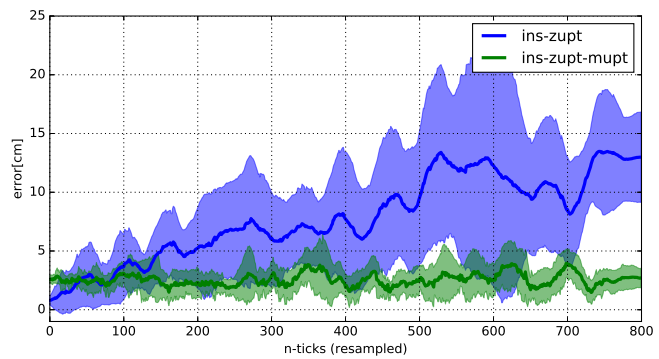

Fig. 5: Position estimation errors.

After prototyping a system with another source of correction the most important thing to check is if it's error does not accumulate over time.

The absolute error (measured as Euclidean distance between the true and estimated path of two methods over time) is depicted on Fig. 5. The error is computed as an average over all 10 samples of different trials along with standard deviation. The most important observation is that that ins-zupt-mupt does not drift over time ( $p<0.01$ using Augmented Dickey-Fuller Test for unit root testing of processes-stationary test), while ins-zupt accumulates errors over time. The trials without zerovelocity phases were not enclosed into the results since its error was too significant.

\section{CONCLUSION AND FUTURE WORKS}

In this paper, position tracking system based on data fusion from inertial measurement unit and positioning in magnetic field was presented. Inertial navigation was performed using the so-called zero-velocity updates and modelled by the means of the Kalman Filter. The common problem with error accumulation of inertial system has been solved by positioning sensor in the presence of static magnetic field.

The presented method enables us to build more robust human-computer interfaces which requires positioning subsystem, because it requires just one sensor for magnetic field sensing. Static magnetic field can be easily generated with no energetic cost by various (in terms of size and strength) neodymium magnets and the magnetometer most of the time is already mounted into IMU unit.

The previous works consisted of array of sensor that was rigidly mounted, which can introduce some problem in reallife systems. Usage of just one sensor, however, introduces some drawbacks: (1) the field of magnet needs to be known precisely in advance, therefore a calibration procedure needs to be introduced in advance (2) from theoretical point of view the additional corrections that are made are not unambiguousthe cost function Eq. 7 minimizes position error with given orientation. The second problem could lead to error in yawangle estimation.

In this paper authors did not investigated orientation estimation errors. Nevertheless, the presented algorithm corrects the orientation estimates in zero-velocity phases. The estimates are sufficiently good for most applications because orientation estimation is less prone to errors since it can be corrected by data fusion filter and needs only one integration. Nevertheless, in future work promising idea is to estimate orientation errors taking the whole path into consideration by forming some lagintroducing corrections. The naive idea is to correct orientation minimizing the magnetic field vector derivation from theoretical model in more than one points, assuming some tuned springiness between the measuring points.

\section{REFERENCES}

[1] F. Ayazi, "Multi-dof inertial mems: From gaming to dead reckoning," in 2011 16th International Solid-State Sensors, Actuators and Microsystems Conference, 2011.

[2] D. E. Serrano, "Design and analysis of mems accelerometers," in IEEE Sensors, 2013

[3] S. O. Madgwick, "An efficient orientation filter for inertial and inertial/magnetic sensor arrays," Report $x$-io and University of Bristol (UK), 2010.

[4] E. Foxlin, "Pedestrian tracking with shoe-mounted inertial sensors," Computer Graphics and Applications, IEEE, vol. 25, no. 6, pp. 38-46,

[5] R. Olsen and A. Farstad, "Electromagnetic direction finding experiments for location of trapped miners," Geoscience Electronics, IEEE Transactions on, vol. 11, no. 4, pp. 178-185, 1973.

[6] F. H. Raab, E. B. Blood, T. O. Steiner, and H. R. Jones, "Magnetic position and orientation tracking system," Aerospace and Electronic Systems, IEEE Transactions on, no. 5, pp. 709-718, 1979.

[7] F. H. Raab, "Quasi-static magnetic-field technique for determining position and orientation," Geoscience and Remote Sensing, IEEE Transactions on, no. 4, pp. 235-243, 1981.

[8] D. Roetenberg, P. J. Slycke, and P. H. Veltink, "Ambulatory position and orientation tracking fusing magnetic and inertial sensing," Biomedical Engineering, IEEE Transactions on, vol. 54, no. 5, pp. 883-890, 2007.

[9] E. R. Bachmann, I. Duman, U. Usta, R. B. Mcghee, X. Yun, and M. Zyda, "Orientation tracking for humans and robots using inertial sensors" in Computational Intelligence in Robotics and Automation, 1999. CIRA'99. Proceedings. 1999 IEEE International Symposium on. IEEE, 1999, pp. 187-194.

[10] R. Zhu and Z. Zhou, "A real-time articulated human motion tracking using tri-axis inertial/magnetic sensors package," Neural Systems and Rehabilitation Engineering, IEEE Transactions on, vol. 12, no. 2, pp. 295-302, 2004

[11] X. Yun, J. Calusdian, E. R. Bachmann, and R. B. McGhee, "Estimation of human foot motion during normal walking using inertial and magnetic sensor measurements," Instrumentation and Measurement, IEEE Transactions on, vol. 61, no. 7, pp. 2059-2072, 2012

[12] P. Robertson, M. Angermann, B. Krach, and M. Khider, "Inertial systems based joint mapping and positioning for pedestrian navigation," in Proc ION GNSS, 2009. 
[13] Ö. Bebek, M. A. Suster, S. Rajgopal, M. J. Fu, X. Huang, M. C. Çavuşoğlu, D. J. Young, M. Mehregany, A. J. Van den Bogert, and C. H. Mastrangelo, "Personal navigation via high-resolution gait-corrected inertial measurement units," Instrumentation and Measurement, IEEE Transactions on, vol. 59, no. 11, pp. 3018-3027, 2010.

[14] J.-O. Nilsson, I. Skog, and P. Händel, "A note on the limitations of zupts and the implications on sensor error modeling," in 2012 International Conference on Indoor Positioning and Indoor Navigation (IPIN), 1315th November 2012, 2012.

[15] G. Zachmann, "Distortion correction of magnetic fields for position tracking," in Computer Graphics International, 1997. Proceedings. IEEE, 1997, pp. 213-220.

[16] R. Alimi, N. Geron, E. Weiss, and T. Ram-Cohen, "Ferromagnetic mass localization in check point configuration using a levenberg marquardt algorithm," Sensors, vol. 9, no. 11, pp. 8852-8862, 2009.

[17] N. Wahlstrom, J. Callmer, and F. Gustafsson, "Magnetometers for tracking metallic targets," in Information Fusion (FUSION), 2010 13th Conference on. IEEE, 2010, pp. 1-8.

[18] W. Weitschies, R. Kötitz, D. Cordini, and L. Trahms, "High-resolution monitoring of the gastrointestinal transit of a magnetically marked capsule," Journal of pharmaceutical sciences, vol. 86, no. 11, pp. 1218 1222, 1997.

[19] V. Schlageter, P.-A. Besse, R. Popovic, and P. Kucera, "Tracking system with five degrees of freedom using a $2 \mathrm{~d}$-array of hall sensors and permanent magnet," Sensors and Actuators A: Physical, vol. 92, no. 1, pp. 37-42, 2001.

[20] X. Wang, M. Q. Meng, and C. Hu, "A localization method using 3axis magnetoresistive sensors for tracking of capsule endoscope," in Engineering in Medicine and Biology Society, 2006. EMBS'06. 28th Annual International Conference of the IEEE. IEEE, 2006, pp. 2522 2525.

[21] J. T. Sherman, J. K. Lubkert, R. S. Popovic, and M. R. DiSilvestro, "Characterization of a novel magnetic tracking system," Magnetics,
IEEE Transactions on, vol. 43, no. 6, pp. 2725-2727, 2007.

[22] H. G. Kortier, J. Antonsson, H. M. Schepers, F. Gustafsson, and P. H. Veltink, "Hand pose estimation by fusion of inertial and magnetic sensing aided by a permanent magnet," Neural Systems and Rehabilitation Engineering, IEEE Transactions on, vol. 23, no. 5, pp. 796-806, 2015.

[23] J. B. M. Mark A. Heald, Classical Electromagnetic Radiation, 3rd ed. Brooks Cole, 1995.

[24] P. J. Green and R. Sibson, "Computing dirichlet tessellations in the plane," The Computer Journal, vol. 21, no. 2, pp. 168-173, 1978.

[25] H. Ledoux, Modelling three-dimensional fields in geoscience with the Voronoi diagram and its dual. University of Glamorgan, 2006.

[26] E. P. Mücke, I. Saias, and B. Zhu, "Fast randomized point location without preprocessing in two-and three-dimensional delaunay triangulations," in Proceedings of the twelfth annual symposium on Computational geometry. ACM, 1996, pp. 274-283.

[27] O. Hallingstad, "Design of a kalman filter for transfer alignment," DTIC Document, Tech. Rep., 1989.

[28] M. Meina, A. Krasuski, and K. Rykaczewski, "Model fusion for inertialbased personal dead reckoning systems," in Sensors Applications Symposium (SAS), 2015 IEEE. IEEE, 2015, pp. 1-6.

[29] A. Peruzzi, U. D. Croce, and A. Cereatti, "Estimation of stride length in level walking using an inertial measurement unit attached to the foot: A validation of the zero velocity assumption during stance," Journal of Biomechanics, vol. 44, no. 10, pp. 1991-1994, 2011. [Online]. Available: http://www.sciencedirect.com/science/article/pii/S0021929011003666

[30] A. R. Jimenez, F. Seco, C. Prieto, and J. Guevara, "A comparison of pedestrian dead-reckoning algorithms using a low-cost mems imu," in Intelligent Signal Processing, 2009. WISP 2009. IEEE International Symposium on, Aug 2009, pp. 37-42.

[31] I. Skog, J. O. Nilsson, and P. Händel, "Evaluation of zero-velocity detectors for foot-mounted inertial navigation systems," in Indoor Positioning and Indoor Navigation (IPIN), 2010 International Conference on, Sept 2010, pp. 1-6. 